\title{
Delayed Epidural Mucin Collection after Surgery for Spinal Metastatic Pancreatic Adenocarcinoma
}

\author{
Dong Ha Kim, \\ Dong Hwan Kim, \\ Hwan Soo Kim, \\ Kyoung Hyup Nam, \\ Byung Kwan Choi, \\ In Ho Han
}

Department of Neurosurgery, Medical Research Institute, Pusan National University Hospital, Busan, Korea

\section{Corresponding Author:}

In Ho Han

Department of Neurosurgery, Pusan National University Hospital, 179 Gudeok-ro, Seo-gu, Busan 49241, Korea

Tel: +82-51-240-7257

Fax: +82-51-244-0282

E-mail: farlateral@hanmail.net

Received: September 22, 2016

Revised: January 17, 2017

Accepted: January 19, 2017
Copyright () 2017 by The Korean Spinal Neurosurgery Society

This is an open access article distributed under the terms of the Creative Commons Attribution Non-Commercial License (http://creativecommons.org/licenses/by-n $\mathrm{c} / 4.0 /$ ) which permits unrestricted non-commercial use, distribution, and reproduction in any medium, provided the original work is properly cited.

A rare case of delayed large epidural mucin collection causing neurologic deficit after surgery for metastatic pancreatic cancer is reported. A 65-year-old man presented with intractable upper-thoracic back pain radiating to the chest and gait disturbance. He had a history of subtotal pancreatectomy due to intraductal papillary mucinous neoplasm (IPMN) of the pancreas and concurrent chemotherapy. Eight months after pancreatectomy, multiple thoracic spinal metastasis was diagnosed with routine up positron emission tomography-computed tomography. Radiotherapy for spinal metastasis and subsequent chemotherapy was carried out. Sixteen months after pancreatectomy, gait disturbance occurred and follow-up thoracic magnetic resonance imaging (MRI) showed aggravation of metastasis at T2 and T4 compressing the spinal cord. We performed a decompressive laminectomy with subtotal resection of the tumor masses and pedicle screw fixation at C7-T6. Neurologic status improved after the operation. Histopathologic examinations revealed the tumor as metastatic mucin producing adenocarcinoma. Three months after surgery, motor weakness and pain was reappeared. MRI showed large amount of epidural fluid collection. We performed wound revision and there was large amount of gelatinous fluid at the epidural space. We suggest that postoperative mucin collection and wound problems should be considered after surgery for mucin producing metastatic pancreatic tumor.

Key Words: Spinal neoplasm, Neoplasm metastasis, Mucins, Adenocarcinoma

\section{INTRODUCTION}

Vertebral column is a common site for bony metastasis in patients with systemic malignancy. But if we consider metastasis of adenocarcinoma, especially vertebral metastasis of intraductal papillary mucinous neoplasm (IPMN) is rare $^{4,7}$. IPMN is a pancreatic neoplasm, characterized by production of mucinous fluid, cystic dilatation of the pancreatic ducts, and intrapapillary growth ${ }^{2,89}$. Mucin could be produced by metastatic spinal tumor originating from pancreatic IPMN as the primary tumor. However, no report has addressed the mucin-associated complication after spinal surgery for metastatic pancreatic cancer.

Here, we report extremely rare case of delayed large epidural mucin collection causing neurologic deficit after surgery for metastatic pancreatic cancer.

\section{CASE REPORT}

A 65-year-old man presented with abdominal discomfort and intractable upper-thoracic back pain radiating to the chest and gait disturbance. He had a history of subtotal pancreatectomy and total splenectomy due to IPMN of the pancreas at another hospital. The diameter of tumor mass was $5.2 \mathrm{~cm}$, and histopathologic finding confirmed that there was invasion of peripancreatic soft tissue and lymph node metastasis. He underwent chemotherapy with gemcitabine hydrochloride as adjuvant therapy. Eight months after subtotal pancreatectomy, no specific symptoms were developed but spinal metastasis was found at T2 and T4 on a first positron emission tomography-computed tomography. Radiotherapy delivering $44 \mathrm{~Gy}$ in 20 fractions was applied to spinal bone metastasis and TS-1 chemotherapy was subsequently implemented. Sixteen months after pancreatectomy, weakness in lower extremities (strength $4 / 5$ ) and gait disturbance were occurred and thoracic magnetic resonance imaging (MRI) showed interval aggravation of metastasis at $\mathrm{T} 2$ and $\mathrm{T} 4$ involving epidural space and posterior element (Fig. 1). We performed total laminectomy and pediculectomy T2 and T4 with subtotal resection of the tumor masses for decompression, and pedicle screw fixation at C7-T6 for stabilization (Fig. 2). After the operation, patient's lower limb motor was nearly intact, and he could walk without support.

Three months after spinal surgery, motor weakness and paresthesia reappeared. MRI showed significant loculated fluid collection 

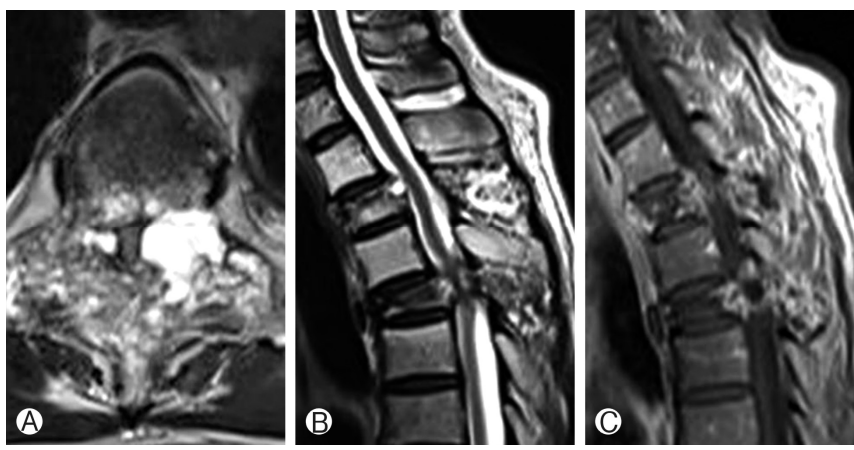

Fig. 1. Axial (A) and sagittal T2- (B) and contrast enhanced T1-weighted $(C)$ magnetic resonance imaging shows vertebral collapse and cord compromise due to metastatic tumors of T2 and T4.

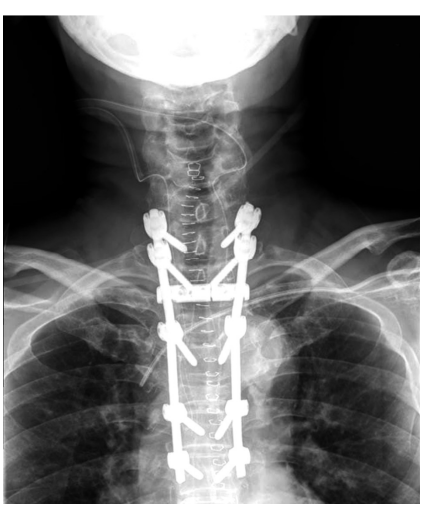

Fig. 2. Postoperative image of total laminectomy and pediculectomy T2 and T4 with subtotal resection of the tumor masses, and pedicle screw fixation at $\mathrm{C} 7-\mathrm{T} 6$.
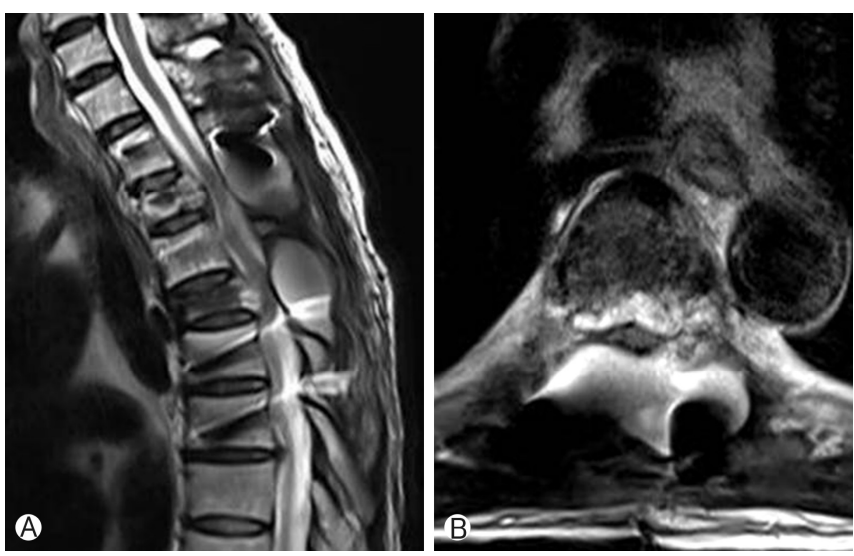

Fig. 3. Sagittal (A) and Axial (B) T2-weighted magnetic resonance imaging of 3 months after the operation shows large amount of accumulated fluid with compressing the spinal cord of T4 at the laminectomy site.

around the laminectomy site which resulted in compression of the spinal cord at the level of T4 (Fig. 3). We performed wound revision and large amount of fluid was expelled out. The nature of the fluid was transparent, mucoid, and sticky (Fig. 4). Total volume of removed fluid was about $150 \mathrm{~mL}$. Liquid based cytology examination showed the fluid component as mucin and tumor

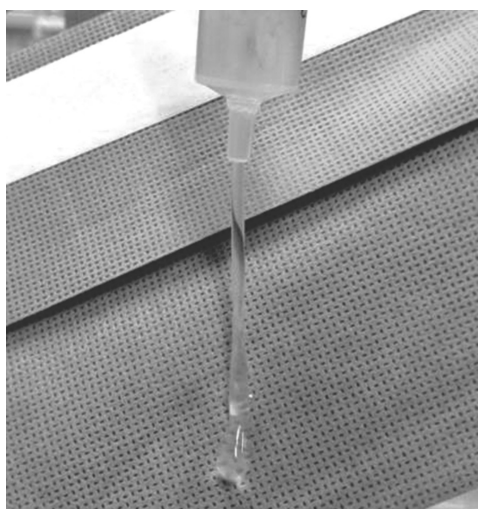

Fig. 4. Gross finding of aspirated fluid showing a mucoid feature.

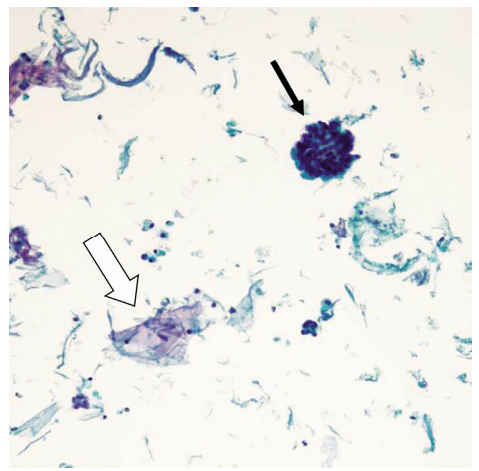

Fig. 5. Liquid based cytology examination showed the fluid component as mucin (open arrow) and adenocarcinoma cells (black arrow) $($ H\&E. $\times 200)$.

cells (Fig. 5). After the revision, motor power of lower extremities was improved, but small amount of mucin was continually expelled through a drainage site of the skin requiring repetitive paracentesis. At that time, patient complained persistent abdominal discomfort, abdominal computed tomography revealed new appearance of carcinomatosis peritonei. Six months after first spine surgery, he expired due to deteriorated pneumonia.

\section{DISCUSSION}

In most cases, IPMN of the pancreas is a slow-growing tumor and shows excellent prognosis ${ }^{4}$. However, invasive IPMN has a high recurrence and high mortality ${ }^{8}$. In particular, lymph node metastasis is an important prognostic factor and frequent in patients with invasive IPMNs ${ }^{7}$. For those patients with invasive IPMNs, 1-, 2-year actuarial survival rates were 45\%, 24\% with positive lymph nodes, on the other hand 95\%, 95\% with negative lymph nodes ${ }^{6}$. In addition, distant metastasis may be a poor prognostic factor ${ }^{4}$. In our case, because of poor prognosis of invasive IPMN with positive lymph node metastasis, we selected subtotal removal of tumor mass as an operative method. Unlike lymph node metastasis, distant metastasis of IPMNs is rare $^{1,7}$. Liver and lung have been reported as the common sites of distant metastasis ${ }^{7,8)}$. In particular, vertebral metastasis of invasive IPMN is extremely rare and only one case has been reported $^{7}$. 
Characteristically, IPMNs of the pancreas produce excessive gelatinous mucin by ductal epithelial cells which may cause biliary obstruction, pancreatitis, and fibrosis ${ }^{5}$. The excessive mucin production of the IPMN can occasionally cause rupture of the pancreatic duct, resulting in spillage of mucinous material into the peritoneal cavity and pseudomxyoma peritonei ${ }^{-5)}$. Metastatic tumor of IPMNs may also produce mucin materials. However, complications associated with excessive mucin collection of metastatic IPMNs have rarely been reported, especially in vertebral metastasis. In our case, subtotal tumor debulking, laminectomy and fixation with pedicle screw insertion were performed in a routine manner ${ }^{3)}$. However, remnant metastatic tumor cells continually produced mucin. Accumulated mucin at the laminectomy site compressed the spinal cord, resulting in neurologic aggravation 3 months after surgery. The current case is the first report of mucin-associated complication of metastatic IPMNs in spine.

No definite guidelines for treatment of extrapancreatic mucin have been reported. In cases of pseudomyxoma peritonei by IPMNs, initial treatment includes radical, repetitive debulking operations with complete mucus removal ${ }^{5)}$. However, radical tumor resection and mucin removal may be limited in metastatic spinal tumor. Beside the radical operation, many alternative treatments have been applied including mucolytic agents, phototherapy, adjuvant systemic chemotherapy, and radiotherapy $y^{5)}$. However, clinical course of pseudomyxoma peritonei ultimately shows an overall poor prognosis ${ }^{4,8}$. In our case, radiotherapy with concurrent chemotherapy was not effective in reducing tumor burden. Instead, repetitive paracentesis was performed after revision surgery in order to prevent spinal cord compression by accumulation of mucin.

\section{CONCLUSION}

We report a rare case of delayed epidural mucin collection after surgery for spinal metastatic pancreatic mucinous adenocarcinoma. The patient maintained the motor function until death. The metastatic IPMN can also produce excessive mucin like primary IPMN. Therefore, spine surgeon should be aware of the possibility of mucin-associated delayed neurologic aggravation after surgery for metastatic IPMNs.

\section{CONFLICT OF INTEREST}

No potential conflict of interest relevant to this article was reported.

\section{REFERENCES}

1. Chiang KC, Yu CC, Chen JR, Huang YT, Huang CC, Yeh CN, et al: Oncocytic-type intraductal papillary mucinous neoplasm (IPMN)-derived invasive oncocytic pancreatic carcinoma with brain metastasis: a case report. World J Surg Oncol 10:138, 2012

2. Fritz S, Warshaw AL, Thayer SP: Management of mucin-producing cystic neoplasms of the pancreas. Oncologist 14:125-136, 2009

3. Jeong JH, Roh SW, Rhim SC, Jeon SR: Effectiveness of surgical decompression and fixation in patients with metastatic spinal cord compression. Korean J Spine 4:201-214, 2007

4. Kang MJ, Jang JY, Lee KB, Chang YR, Kwon W, Kim SW: Longterm prospective cohort study of patients undergoing pancreatectomy for intraductal papillary mucinous neoplasm of the pancreas: implications for postoperative surveillance. Ann Surg 260:356-363, 2014

5. Rosenberger LH, Stein LH, Witkiewicz AK, Kennedy EP, Yeo CJ: Intraductal papillary mucinous neoplasm (IPMN) with extra-pancreatic mucin: a case series and review of the literature. J Gastrointest Surg 16:762-770, 2012

6. Sohn TA, Yeo CJ, Cameron JL, Hruban RH, Fukushima N, Campbell $\mathrm{KA}$, et al: Intraductal papillary mucinous neoplasms of the pancreas: an updated experience. Ann Surg 239:788-797, 2004

7. Takuma Y, Kawai D, Makino Y, Saito S, Tanaka S, Ogata M, et al: Vertebral metastasis of intraductal papillary mucinous tumor of the pancreas. Pancreas 33:206-208, 2006

8. Turrini O, Waters JA, Schnelldorfer T, Lillemoe KD, Yiannoutsos CT, Farnell MB, et al: Invasive intraductal papillary mucinous neoplasm: predictors of survival and role of adjuvant therapy. HPB (Oxford) 12:447-455, 2010

9. Whang EE, Danial T, Dunn JC, Ashley SW, Reber HA, Lewin TJ, et al: The spectrum of mucin-producing adenocarcinoma of the pancreas. Pancreas 21:147-151, 2000 